\title{
Magnetic Properties and Magnetocaloric Effect of $\mathrm{DyNi}_{4} \mathrm{Si}$
}

\author{
K. Synoradzki ${ }^{a}$, W. Kowalski ${ }^{a, *}$, M. FAlkowski $^{a, b}$, T. Toliński ${ }^{a}$, A. KowalczyK $^{a}$ \\ ${ }^{a}$ Institute of Molecular Physics, Polish Academy of Sciences, M Smoluchowskiego 17, 60-179 Poznań, Poland \\ ${ }^{b}$ Physics Department, University of Johannesburg, PO Box 524, Auckland Park 2006, South Africa
}

\begin{abstract}
The magnetic properties and magnetocaloric effect (MCE) in $\mathrm{DyNi}_{4} \mathrm{Si}$ have been studied by the magnetization and specific heat measurements. The saturation magnetic moment in $\mu_{0} H=9 \mathrm{~T}$ is equal to $7.9 \mu_{B} /$ f.u at $4.2 \mathrm{~K}$. A significant $\mathrm{MCE}$ has been observed around the second order phase transition at $T_{\mathrm{C}}=11 \mathrm{~K}$. The maximum magnetic entropy change is $-\Delta S_{M}=14.5 \mathrm{~J} \mathrm{~kg}^{-1} \mathrm{~K}^{-1}$ for the magnetic field change of $9 \mathrm{~T}$.
\end{abstract}

DOI: $10.12693 /$ APhysPolA.126.162

PACS: 71.20.Lp, 75.30.Sg, 75.40.Cx, 81.20.Ev

\section{Introduction}

$\mathrm{DyNi}_{4} \mathrm{Si}$ crystallizes in the hexagonal $\mathrm{CaCu}_{5}$-type structure (space group P6 $/ \mathrm{mmm}$ ). At $T_{\mathrm{C}}=11 \mathrm{~K}$ a second order phase transition to a ferromagnetic order has been observed. The heat capacity measurements have shown that $\mathrm{DyNi}_{4} \mathrm{Si}$ is characterized by the enhanced electronic specific heat coefficient $\gamma=72 \mathrm{~mJ} / \mathrm{mol} / \mathrm{K}^{2}$. In the low-temperature dependence of $C_{p}(T)$ a well defined $\lambda$-type anomaly appears near $T_{\mathrm{C}}$ for zero magnetic field. The externally applied magnetic fields lead to the quenching and shifting of the peak in the vicinity of $T_{\mathrm{C}}$ to higher temperatures, typical of ferromagnets [1]. Recently, we have studied the magnetocaloric effect (MCE) in the $\mathrm{NdNi}_{4} \mathrm{Si}$ [2] and $\mathrm{GdNi}_{4} \mathrm{Si}$ [3] compounds. The present investigations concern MCE in the isostructural $\mathrm{DyNi}_{4} \mathrm{Si}$. Apart from the measurements on the bulk sample, we have additionally performed a detailed studies of the effect of the grains size on the maximum value and the temperature dependence of MCE. The modification of the grains size has been obtained by mechanical milling. The sizes of the grains have been checked after various milling times by analysis of the X-ray diffraction (XRD) measurements. A decrease and broadening of MCE has been observed.

\section{Results and discussion}

$\mathrm{DyNi}_{4} \mathrm{Si}$ has been prepared by induction melting of the constituent elements under an argon atmosphere. The samples of different grains size were obtained by mechanical milling performed in a conventional horizontal ball mill rotated at a speed of $80 \mathrm{rev} / \mathrm{min}$. Stainless steel vial and balls were used. The heat capacity measurements were carried out by the relaxation method (two- $\tau$ model) using the commercial Quantum Design Physical Property Measurement System (PPMS). The evolution of the grains size with the milling time has been monitored after each milling cycle by XRD, with the grains

\footnotetext{
*corresponding author; e-mail: pewk@ifmpan.poznan.pl
}

size estimated from the standard Scherrer's formula. Figure 1 shows the XRD patterns after successive milling times. A rather slow decrease and broadening of the Bragg peaks has been observed.

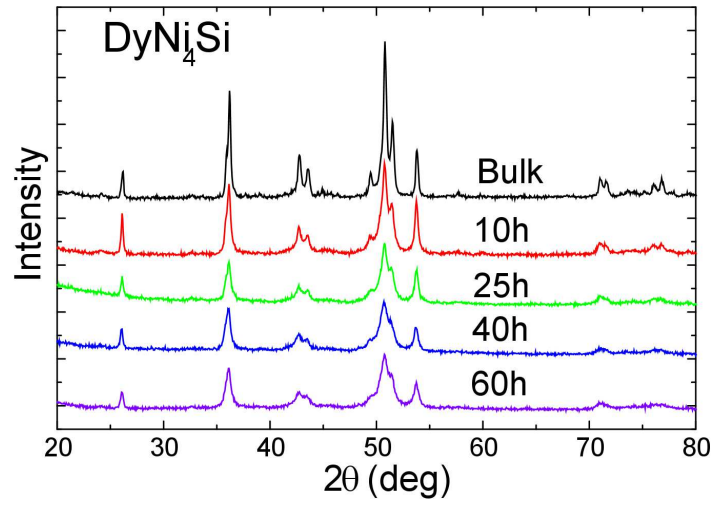

Fig. 1. XRD patterns of the $\mathrm{DyNi}_{4} \mathrm{Si}$ compound for increasing milling time length.

MCE has been determined from the $C_{p}(T)$ data as a function of temperature in constant magnetic field. The magnetic entropy change can be calculated by using the expression:

$$
\Delta S_{M}(T, H)=\int_{0}^{T}\left(\frac{C_{p}(T, H)-C_{p}(T, 0)}{T}\right) \mathrm{d} T,
$$

where $C_{p}(T, H)$ and $C_{p}(T, 0)$ are the values of the specific heat measured in a field $H$ and in zero field. Figure 2 presents MCE obtained from the $C_{p}(T)$ data for a few selected magnetic fields and milling times up to $60 \mathrm{~h}$. For the bulk sample the maximum values of $-\Delta S_{M}$ at the magnetic ordering temperature and for magnetic field changes of 9,5 , and $1 \mathrm{~T}$ are $14.5 \mathrm{~J} \mathrm{~kg}^{-1} \mathrm{~K}^{-1}$, 11.3 $\mathrm{J} \mathrm{kg}^{-1} \mathrm{~K}^{-1}$, and $3.6 \mathrm{~J} \mathrm{~kg}^{-1} \mathrm{~K}^{-1}$, respectively. Next, the isoentropic temperature change $\Delta T_{a d}$ is defined as:

$$
\Delta T_{a d}(T)_{\Delta H}=\left[T(S)_{H}-T(S)_{H=0}\right]_{S} .
$$

Figure 3 shows the temperature dependence of $\Delta T_{a d}$ for similar magnetic field changes and milling times as in the case of $\Delta S_{M}$. The MCE of $\mathrm{DyNi}_{4} \mathrm{Si}$ exhibits similar characteristic values as the compound $\mathrm{GdNi}_{4} \mathrm{Si}$ [3] and better than the $\mathrm{NdNi}_{4} \mathrm{Si}[2]$. 


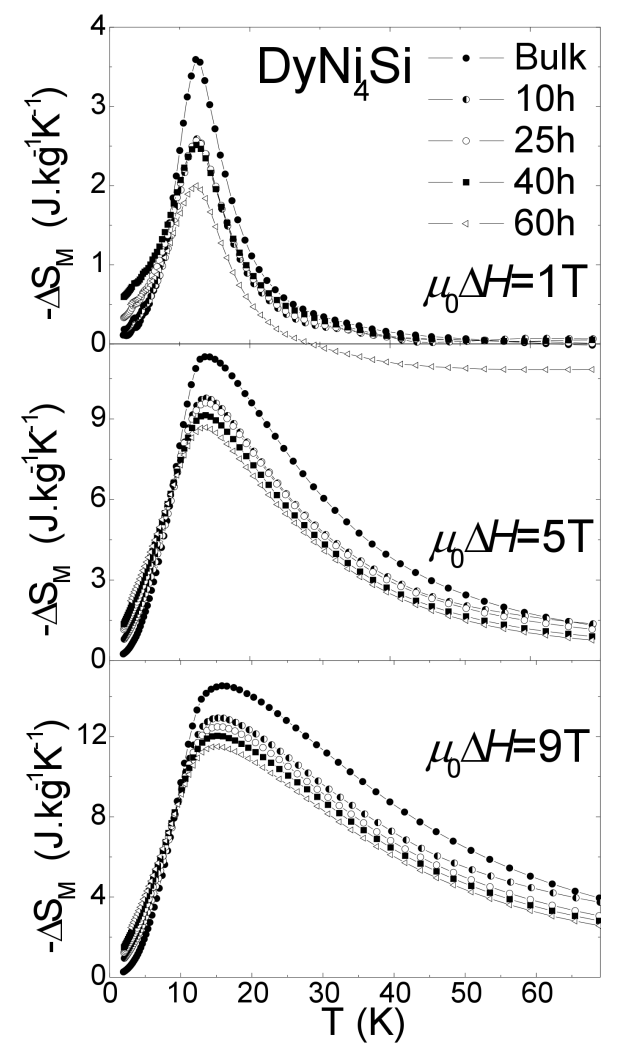

Fig. 2. The magnetic entropy change $-\Delta S_{M}$ for various milling times and magnetic field changes.

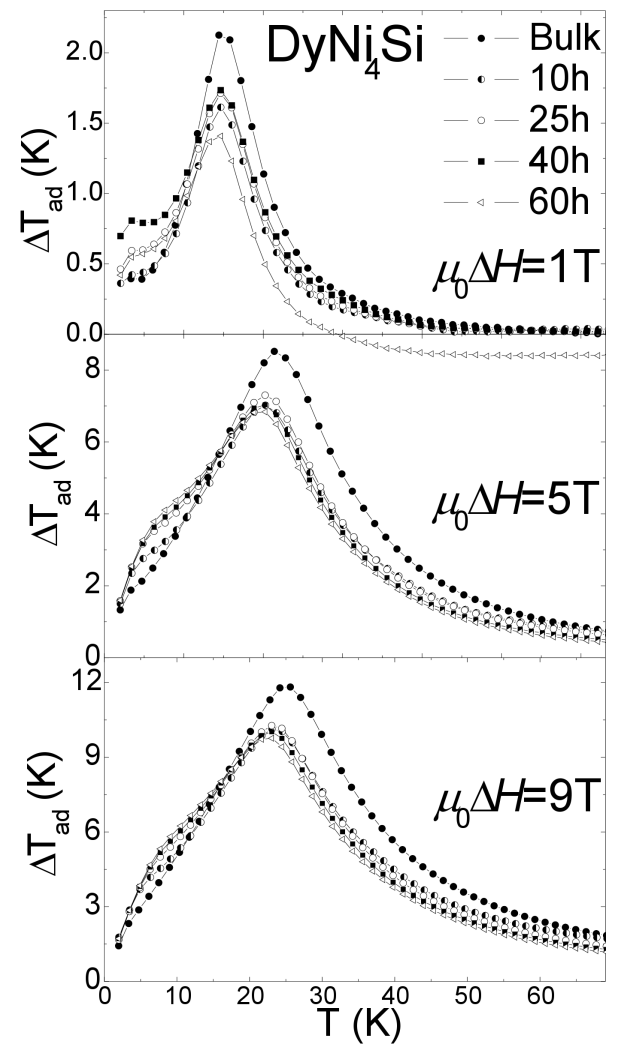

Fig. 3. The adiabatic temperature change $\Delta T_{a d}$ for various milling times and magnetic field changes.
It appears that even mechanical milling time as long as $60 \mathrm{~h}$ does not diminish significantly the magnetocaloric effect, which results obviously from a small reduction of the grains size. It is better evidenced by the dependence of relative cooling power $\left(\mathrm{RCP}_{S}\right)$ on the milling time, displayed in Fig. 4. The $\mathrm{RCP}_{S}$ is one of parameters used to describe the figure of merit of the MCE materials. It is defined as a product of the maximum value and the full width at half maximum of the $\Delta S_{M}(T)$ curves. It results from Fig. 4, that only at the beginning of the milling a drop of $\mathrm{RCP}_{S}$ occurs and then it proceeds slowly. The grain size of the bulk material is not shown in the inset of Fig. 4 because it has shown a large spread of the values for different Bragg peaks.

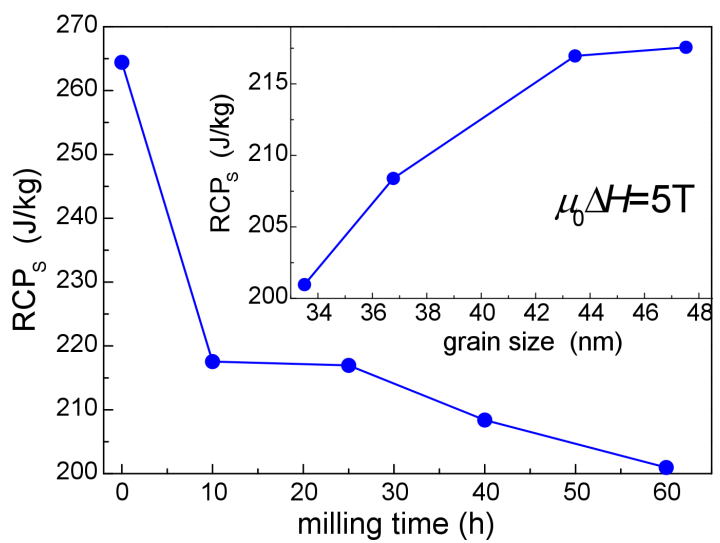

Fig. 4. Relative cooling power $\mathrm{RCP}_{S}$ as a function of the grain size and the milling time.

\section{Conclusions}

We have investigated the magnetocaloric effect in $\mathrm{DyNi}_{4} \mathrm{Si}$ on the basis of the specific heat measurements. It exhibits relatively large values of the isothermal entropy change $-\Delta S_{M}$ and the isoentropic temperature change around the magnetic ordering temperature. It has been shown that apart from the first stage of the process, the influence of the mechanical milling on the grains size and the MCE parameters is not strong.

\section{Acknowledgments}

This work was supported by the funds of the National Science Centre as a research project no. N N507 219540 in years 2011-2013.

\section{References}

[1] M. Falkowski, A. Kowalczyk, M. Reiffers, T. Toliński, E. Gažo, M. Zapotoková, G. Chełkowska, IEEE Trans. Magnet. 44, 3056 (2008).

[2] M. Falkowski, T. Toliński, A. Kowalczyk, Acta Phys. Pol. A 121, 1290 (2012).

[3] T. Toliński, M. Falkowski, K. Synoradzki, A. Hoser, N. Stüßer, J. Alloys Compd. 523, 43 (2012). 